\title{
Formation of electron energy spectra during magnetic reconnection in laser-produced plasma
}

Kai Huang ${ }^{1,2}$, Quanming $\mathrm{Lu}^{1,2}$, Can Huang ${ }^{1,2}$, Quanli Dong ${ }^{3,6}$, Huanyu Wang ${ }^{1,2}$, Feibin Fan $^{1,2}$, Zhengming Sheng ${ }^{4,5,6}$, Shui Wang ${ }^{1,2}$, and Jie Zhang ${ }^{4,6}$

${ }^{1}$ CAS Key Lab of Geospace Environment, Department of Geophysics and Planetary Science, University of Science and Technology of China, Hefei 230026, China

${ }^{2}$ Collaborative Innovation Centers of Astronautical Science and Technology, Harbin, China

${ }^{3}$ School of Physics and Optoelectronic Engineering, Ludong University, Yantai 264025, China

${ }^{4}$ MoE Key Laboratory for Laser Plasmas and School of Physics and Astronomy, Shanghai Jiao Tong University, Shanghai 200240, China

${ }^{5}$ SUPA, Department of Physics, University of Strathclyde, Glasgow G4 0NG, UK

${ }^{6}$ Innovative Collaboration Center of IFSA, Shanghai Jiao Tong University, Shanghai 200240, China

Corresponding Author: Quanming Lu

Email: qmlu@ustc.edu.cn 


\section{ABSTRACT}

The energetic electron spectra formed during magnetic reconnection between two laser-produced plasma bubbles are investigated by use of two-dimensional particle-in-cell simulations. It is found that the evolution of such interaction between the two plasma bubbles can be separated into two distinct stages: the squeezing and reconnection stages. In the squeezing stage, when the two plasma bubbles expand quickly and collide with each other, the magnetic field in the inflow region is greatly enhanced. In the second stage, a thin current sheet is formed between the two plasma bubbles, and then magnetic reconnection occurs therein. During the squeezing stage, electrons are heated in the perpendicular direction by betatron acceleration due to the enhancement of the magnetic field around the plasma bubbles. Meanwhile, non-thermal electrons are generated by the Fermi mechanism when these electrons bounce between the two plasma bubbles approaching quickly and get accelerated mainly by the convective electric field associated with the plasma bubbles. During the reconnection stage, electrons get further accelerated mainly by the reconnection electric field in the vicinity of the $\mathrm{X}$ line. When the expanding speed of the plasma bubbles is sufficiently large, the formed electron energy spectra have a kappa distribution, where the lower energy part satisfies a Maxwellian function and the higher energy part is a power-law distribution. Moreover, the increase of the expanding speed will result in the hardening of formed power-law spectra in both the squeezing and reconnection stages. 


\section{INTRODUCTION}

Magnetic reconnection is a fundamental process allowing topological change of magnetic field lines and rapid conversion of magnetic energy into plasma kinetic and thermal energy. It is suggested that many kinds of explosive phenomena in plasma such as solar flares, ${ }^{1-2}$ magnetosphere substorms ${ }^{3-5}$ and sawtooth crash in tokamaks ${ }^{6,7}$ are related to magnetic reconnection. The production of energetic electrons is also a significant feature of magnetic reconnection. For example, in solar flares, up to $50 \%$ of energy release can appear as energetic electrons, ${ }^{8,9}$ which in general have a power-law spectrum. Electron acceleration during magnetic reconnection has been extensively studied in recent years. Electrons can be accelerated by the reconnection electric field around the $\mathrm{X}$ line,${ }^{10-15}$ as well as in the jet front by the betatron and Fermi mechanisms. ${ }^{16-19}$ Magnetic islands have also been considered to play an important role in electron acceleration during magnetic reconnection. Electrons can get accelerated when they are trapped and reflected at the two ends of an island, ${ }^{11,20-21}$ as well as at the merging region when two islands begin to coalesce, ${ }^{22-25}$ while both simulations and observations have shown that the coalescence of magnetic islands should be a common phenomenon during magnetic reconnection. ${ }^{26-29}$ Although much effort has been made to understand the process of electron acceleration during magnetic reconnection, how a power-law spectrum of energetic electrons is generated is still a mystery. ${ }^{30}$

Recently, several experiments of magnetic reconnection in high-energy-density (HED) laser-produced plasma have been conducted at laser facilities Vulcan, ${ }^{31-33}$ OMEGA $^{34,35}$ and Shenguang-II. ${ }^{36,37}$ In these experiments, two pulses of high power laser beams are focused on a planar foil target, the target is suddenly ionized and two plasma bubbles are formed. The two plasma bubbles expand at a supersonic speed, and the toroidal magnetic field is generated around the plasma bubbles. The two plasma bubbles collide with each other and magnetic reconnection occurs at the 
squeezing region. Different from magnetic reconnection in a Harris current sheet, before development of the reconnection of toroidal magnetic field lines associated with the two laser-produced plasma bubbles, the magnetic field in the inflow region is greatly enhanced due to the squeezing of the two plasma bubbles, ${ }^{38-40}$ which are expanding with a speed much higher that the Alfven speed.

Both experiments and particle-in-cell (PIC) simulations have demonstrated the production of energetic electrons during magnetic reconnection in laser-produced plasma, and usually these electrons have power-law spectrum. ${ }^{37,41}$ By performing two-dimensional (2-D) PIC simulations, $\mathrm{Lu}$ et $a \mathrm{l}^{42}$ investigated the process of electron acceleration during magnetic reconnection in laser-produced plasma, and found that besides the reconnection electric field, Fermi and betatron mechanisms also play an important role during the expanding stage. The electrons in the magnetic ribbons are energized through betatron mechanism due to the enhancement of the magnetic field, while Fermi mechanism takes effect when electrons are trapped and bounced repeatedly between the two expanding bubbles. However, it is still in debate regarding how a power-law spectrum of energetic electrons is formed during laser-produced plasma. In this paper, with the use of 2-D PIC simulations, we investigate what the roles of the reconnection electric field, betatron and Fermi mechanisms play in the formation of the electron energy spectra during magnetic reconnection in laser-produced plasma.

This paper is organized as follow. In section 2, we describe the simulation model. In section 3, the simulation results are presented. Finally, conclusions and discussion are given in section 4 .

\section{SIMULATION MODEL}

In our 2-D PIC simulation model, the electromagnetic field is defined on the grids and updated by solving Maxwell's equations with a full explicit algorithm. Ions and electrons are relativistically advanced in the electromagnetic field. The 
laser-plasma interaction and generation process of the magnetic field are not involved in our simulations, and the initial conditions in the simulations are set corresponding to the plasma bubble expanding phase in the experiment. The conditions are similar to those in previous works: ${ }^{38-40}$ two circular plasma bubbles are defined in $(x, y)$ plane with the toroidal magnetic field around them. The simulation domain size is $\left[-L_{x}, L_{x}\right] \times\left[-L_{y}, L_{y}\right]$, and the centers of the two plasma bubbles are $\left(0,-L_{0}\right)$ and $\left(0, L_{0}\right)$. The radius vectors from the center of each plasma bubble are defined as $\mathbf{r}^{(1)}=\left(x, y+L_{0}\right)$ and $\mathbf{r}^{(2)}=\left(x, y-L_{0}\right)$.

There are two kinds of magnetic field geometry in our simulations: AP case (with reconnection) and $\mathrm{P}$ case (without reconnection). The magnetic field is initialized as the sum of two toroidal ribbons $\mathbf{B}^{(1)}+\mathbf{B}^{(2)}$. In AP case, the magnetic field is defined as:

$$
\mathbf{B}^{(i)}(\mathbf{r})=\left\{\begin{array}{cc}
\mathbf{B}_{0} \sin \left(\frac{\pi\left(L_{n}-r^{(i)}\right)}{2 L_{B}}\right) \hat{\mathbf{r}}^{(i)} \times \hat{\mathbf{z}} & r^{(i)} \in\left[\begin{array}{c}
\left.L_{n}-2 L_{B}, L_{n}\right] \\
\text { otherwise }
\end{array}\right. \\
0 & \text { cthers }
\end{array}\right.
$$

Here, $L_{n}$ is the radius of the two bubbles, and $L_{n}<\min \left\{L_{0}, L_{x}\right\}, L_{0}+L_{n}<L_{y}$, so the simulation domain can contain two whole bubbles. $L_{B}$ is the half width of the initial magnetic ribbons. In $\mathrm{P}$ case, the magnetic field is

$$
\mathbf{B}^{(i)}(\mathbf{r})=\left\{\begin{array}{cc}
\mathbf{B}_{0} \sin \left(\frac{\pi\left(L_{n}-r^{(1)}\right)}{2 L_{B}}\right) \hat{\mathbf{r}}^{(1)} \times \hat{\mathbf{z}} & r^{(1)} \in\left[L_{n}-2 L_{B}, L_{n}\right] \\
0 & \text { otherwise } \\
-\mathbf{B}_{0} \sin \left(\frac{\pi\left(L_{n}-r^{(2)}\right)}{2 L_{B}}\right) \hat{\mathbf{r}}^{(2)} \times \hat{\mathbf{z}} & r^{(2)} \in\left[L_{n}-2 L_{B}, L_{n}\right]
\end{array}\right.
$$

An initial out-of-plane current is added to satisfy the Ampere's Law. The initial density is $n_{b}+n^{(1)}+n^{(2)}$, where $n_{b}$ is the background density and $n^{(i)}(i=1,2)$ is the density of each bubble, which is defined as: 


$$
n^{(i)}(\mathbf{r})=\left\{\begin{array}{cc}
\left(n_{0}-n_{b}\right) \cos ^{2}\left(\frac{\pi r^{(i)}}{2 L_{n}}\right) & r^{(i)}<L_{n} \\
0 & \text { otherwise }
\end{array}\right.
$$

Where $n_{0}$ is the peak density of the bubbles, and we choose $n_{b}=0.1 n_{0}$. The initial expanding velocity of the plasma bubbles is $\mathbf{V}^{(1)}+\mathbf{V}^{(2)}$, and $\mathbf{V}^{(i)}(i=1,2)$ is

$$
\mathbf{V}^{(i)}(\mathbf{r})=\left\{\begin{array}{cc}
V_{0} \sin \left(\frac{\pi r^{(i)}}{L_{n}}\right) \hat{\mathbf{r}}^{(i)} & r^{(i)}<L_{n} \\
0 & \text { otherwise }
\end{array}\right.
$$

where $V_{0}$ is the initial expanding speed of the two plasma bubbles.

To be consistent with the plasma flow, an initial electric field $\mathbf{E}=-\mathbf{V} \times \mathbf{B}$ is imposed. The expansion speed of the plasma bubbles is usually supersonic, and in experiments the sonic Mach number is $M_{s}=V_{0} / c_{s}=2-8{ }^{43,} 44$ (where $c_{s}\left(=\sqrt{\gamma Z T_{e} / m_{i}}\right)$ is the sound speed, and $Z$ is the ion charge).To study the influence of the expansion speed on the electron energy spectrum, we run 5 sub cases for both $\mathrm{AP}$ case and $\mathrm{P}$ case. The expansion speeds of the five cases are set to be $V_{0} / c_{s}=2,3$, 4,5 , and 6. The sound speed $c_{s}$ is equal to $1.545 v_{A}$ (where $v_{A}$ is the Alfven speed based on $n_{0}$ and $B_{0}$ ) in our simulations.

The size of the plasma bubbles is $L_{n}=12 d_{i}$ in our simulations and $L_{B}=2 d_{i}$, where $d_{i}=c / \omega_{p i}$ is the ion inertial length based on $n_{0} . L_{0}$ is set to be $12.5 d_{i}$. The ion-to-electron mass ratio $\mathrm{Zm} m_{i} / m_{e}$ is 100 , and the light speed is $c=75 v_{A}$. The initial temperatures of ions and electrons are set to be uniform $T_{i}=T_{e}=0.025 m_{e} c^{2}$, and their initial velocity distributions are Maxwellian with the bulk velocities in the radial direction and drift velocities in the $z$ direction to supply the out-of-plane current. The plasma beta is $\beta_{e}=\beta_{i}=2 \mu_{0} n_{0} k_{B} T_{e} / B_{0}^{2} \approx 2.9$. In the simulations, 
$L_{x}=25 d_{i}$ and $L_{y}=35 d_{i}$, and the number of grid point is $N_{x} \times N_{y}=1000 \times 1400$ with a spatial resolution of $\Delta x=\Delta y=0.05 d_{i}$. The time step is $\Delta t=0.0001 \Omega_{i}^{-1}$, where $\Omega_{i}=e B_{0} / m_{i}$ is the ion gyrofrequency. 2560 particles per species in a grid for $n_{0}$ is employed in the simulations. The periodic boundary conditions for both the $\mathrm{x}$ and y directions are used.

\section{SIMULATION RESULTS}

Let us at first analyze the evolution of the interaction between two plasma bubbles expanding with a supersonic speed. Figure 1 shows the magnetic field $B / B_{0}$, and the out-of-plane electron current density $J_{e z} / e n_{0} v_{A}$ at $\Omega_{i} t=0,0.3,0.55$ and 0.75 for (a) AP and (b) P cases, here the expanding speed is $V_{0} / c_{s}=5$. Initially, the evolution of AP case is similar to that of $\mathrm{P}$ case. The two plasma bubbles expand with a supersonic speed and approach together quickly. At the same time, the magnetic field surrounding the plasma bubbles is greatly enhanced due to the expansion of the plasma bubbles, especially in the inflow region where the plasma bubbles suffers a strong squeezing. However, in AP case, a thin electron current sheet is formed between the two plasma bubbles at about $\Omega_{i} t=0.55$, and then at about $\Omega_{i} t=0.58$ the reconnection of magnetic field lines associated with the two plasma bubbles occurs. Therefore, the interaction between the two plasma bubbles in the AP system can be roughly divided into two stages, i.e., the squeezing stage and the reconnection stage. While in $\mathrm{P}$ case, we can only observe the squeezing between the two plasma bubbles. The two stages of the interaction between the two plasma bubbles in AP case with $V_{0} / c_{s}=5$ can be distinguished more clearly in Figure 2, where the time evolution of reconnected magnetic flux $\Phi_{B} / B_{0} d_{i}$, the maximum of upstream magnetic field $B_{\max } / B_{0}$, and the reconnection electric field $E_{z} / v_{A} B_{0}$ at 
$(x, y)=(0,0)$ are plotted. The evolution of the maximum of upstream magnetic field $B_{\max } / B_{0}$ in $\mathrm{P}$ case with $V_{0} / c_{s}=5$ is also shown for reference. Before about $\Omega_{i} t=$ 0.45, the maximum of upstream magnetic field in AP case is almost the same as that in P case. It means that during this time period there is only squeezing between the two plasma bubbles, and no reconnection occurs. The maximum of upstream magnetic field reached its peak at about $\Omega_{i} t=0.6$ in AP case, while in $\mathrm{P}$ case the peak is attained at about $\Omega_{i} t=0.75$. In AP case, at about $\Omega_{i} t=0.6$ the pileup rate of the magnetic flux is equal to the reconnection rate, and about only $25 \%$ of the total magnetic flux has been reconnected. Therefore, in AP case, we can use the time when the maximum of upstream magnetic field reaches its peak to separate the squeezing stage and the reconnection stage. Before that time, the interaction of two plasma bubbles is dominated by the squeezing, while magnetic reconnection becomes important only after $\Omega_{i} t=0.6$. Similar results are obtained in Ref. [45].

Figure 3 describes the electron energy spectra at different times for different expanding speeds $V_{0} / c_{s}=2,3,4,5$, and 6 . Here, $\Omega_{i} t=1.2,0.95,0.75,0.6$ and 0.53 correspond to the times when the maximum of upstream magnetic field reaches its peak in AP cases for $V_{0} / c_{s}=2,3,4,5$, and 6, respectively, while $\Omega_{i} t=1.7,1.2,0.9$, 0.79 and 0.65 correspond to the times when the flux of non-thermal electrons reaches its peak in AP cases for $V_{0} / c_{s}=2,3,4,5$, and 6 , respectively. In order to compare the results in AP cases with those in $\mathrm{P}$ cases, the electron energy spectra at the same times in $\mathrm{P}$ cases as those in AP cases are also plotted in the figure. Here, when we calculate the electron spectra, only the electrons in the ribbons of plasma bubbles $\left(r^{(i)} \in\left[L_{n}-2 L_{B}, L_{n}\right]\right)$ are included. The reason for this is that during magnetic reconnection experiment in laser-driven plasma the background plasma is usually negligible and the plasma in the center of plasma bubbles is not involved in magnetic reconnection. Also, when an electron leaves the boundary of simulation domain, its 
energy will be kept unchanged during the calculation of electron energy spectra. In both AP and P cases, we can find that the formed electron energy spectra satisfy the kappa distribution when the expanding speed is sufficiently large. In the lower energy part, the electron energy spectra satisfy the Maxwellian distribution, where the temperature increases with the increase of the expanding speed; in the higher energy part, the electron energy spectra satisfy the power-law distribution, which becomes harder and harder with the increase of the expanding speed. The electron energy spectra in the squeezing stage of AP cases are similar to those at the corresponding times of P cases. In AP cases, the indices of power-law distribution in the higher energy are 7.3, 6.9 and 5.5 for $V_{0} / c_{s}=4,5$ and 6 , respectively, while they are 8.2, 6.2 and 5.9 in P cases. It means that at the squeezing stage of AP cases the mechanism of electron acceleration is similar to that in P cases. Electrons can be further accelerated at the reconnection stage of AP cases with the increase of energetic electron flux, and the electron energy spectra are harder than those at the corresponding times of $\mathrm{P}$ cases. In AP cases, the indices of power-law distribution in higher energy are 5.7, 6.4 and 5.7 for $V_{0} / c_{s}=4,5$ and 6 , respectively, while they are 9.4, 7.5 and 6.3 in $\mathrm{P}$ cases. Therefore, at the reconnection stage of AP cases, the reconnection electric field plays an important role in electron acceleration, and with the increase of the expanding speed the reconnection electric field become less and less important.

Detailed analysis shows that at the squeezing stage of AP cases the electron parallel temperature changes little, while the corresponding perpendicular temperature increases. Figure 4 shows the electron perpendicular temperature in AP cases versus the average magnetic field in the ribbon of plasma bubbles for different expanding speeds $V_{0} / c_{s}=2,3,4,5$, and 6 . The perpendicular temperature increases with the increase of the expanding speed, and it almost satisfies that $T_{\perp} / B$ is a constant. Therefore, we can conclude that at the squeezing stage of AP cases, the increase of electron temperature is caused by the betatron mechanism, where magnetic momentum is a constant. At the same time, we also find that the increase of electron 
temperature in $\mathrm{P}$ cases is also caused by the betatron mechanism.

Figure 5 shows a typical electron trajectory at the squeezing stage of AP case with $V_{0} / c_{s}=5$, (a) the electron position in the simulation domain, (b) the electron energy versus the electron position in the $y$ direction, (c) the time evolution of electron energy, the works done by the convective electric field $-\mathbf{u}_{i} \times \mathbf{B}$ and non-ideal electric field $\mathbf{E}+\mathbf{u}_{i} \times \mathbf{B}$, and (d) the electron position in the $(y, z)$ plane, where the $z$ position is calculated by $\int v_{z} d t$. Obviously, the electron bounces between the two plasma bubbles approaching quickly, and during this time period the electron moves about 10 ion inertial lengths along the $z$ direction. Every time the electron gets accelerated when it is reflected by the plasma bubbles, and it is accelerated mainly by the work done by the convective electric field. Therefore, we can conclude that the energetic electrons at the squeezing stage of AP cases are accelerated by Fermi mechanism. For comparison, Figure 6 shows a typical electron trajectory in $\mathrm{P}$ case with $V_{0} / c_{s}=5$. Similar to that at the squeezing stage of AP case, the electron suffers Fermi acceleration when it bounces between the two plasma bubbles. The difference is that in $\mathrm{P}$ case the electron only moves about 2 ion inertial lengths along the $z$ direction.

Figure 7 shows initial electron energy distributions, where the energy of these electrons exceeds $0.8 m_{e} c^{2}$ at $\Omega_{i} t=0.6$ for AP and $\mathrm{P}$ cases with $V_{0} / c_{s}=5$. We can find that the initial electron energy distributions in both cases are similar, only the electrons with the initial energy higher than about $0.03 m_{e} c^{2}$ (corresponding to an electron with a velocity of $10 v_{A} \approx 6.5 c_{s}$, larger than the expanding speed $V_{0} / c_{s}=5$ ) can be accelerated to very high energy by the Fermi mechanism.

Figure 8 plots a typical electron trajectory, which is accelerated at the 
reconnection stage of $\mathrm{AP}$ case with $V_{0} / c_{s}=5$, (a) the electron position in the simulation domain, (b) the electron energy versus the electron position in the $y$ direction, (c) the time evolution of electron energy, the works done by the convective electric field $-\mathbf{u}_{i} \times \mathbf{B}$ and non-ideal electric field $\mathbf{E}+\mathbf{u}_{i} \times \mathbf{B}$, and (d) the electron position in the $(y, z)$ plane, where the $z$ position is calculated by $\int v_{z} d t$. At about $\Omega_{i} t=0.6$, the electron enters the diffusion region, and is accelerated by the non-ideal electric field. Different from that in the Fermi acceleration, the work done by the convective electric field is negligible to accelerate electron. It is noted that the electron moves about 10 ion inertial lengths along the $z$ direction, close to that in the Fermi process. Both the Fermi mechanism and reconnection electric field should play important roles to accelerate electrons in three-dimensional situation.

Figure 9 shows the electron energy distributions at $\Omega_{i} t=0.6$, where the energy of these electrons exceeds $1.0 m_{e} c^{2}$ at $\Omega_{i} t=0.79$ for $\mathrm{AP}$ and $\mathrm{P}$ cases with $V_{0} / c_{s}=5$. There are essential differences between AP and P cases. In P cases, the electrons only suffer the Fermi acceleration. In AP case, the energy of most these electrons at $\Omega_{i} t=$ 0.6 is lower than $0.8 m_{e} c^{2}$, and there is no energy threshold for electrons to be accelerated to higher than $1.0 m_{e} c^{2}$ at $\Omega_{i} t=0.79$. Only a small part of these electrons have energy higher than $0.8 m_{e} c^{2}$ at $\Omega_{i} t=0.6$. It means that most of these energetic electrons only suffer acceleration by reconnection electric field, and only a small part are accelerated by both Fermi and reconnection electric field.

\section{CONCLUSIONS AND DISCUSSION}

In this paper, we investigated the evolution of electron energy spectra during magnetic reconnection in laser-produced plasma by use of 2-D PIC simulations. The interaction between the two plasma bubbles expanding with supersonic speed can be 
divided into the squeezing and reconnection stages. During the squeezing stage, the magnetic field in the inflow region is enhanced due to the collision between the two plasma bubbles, and electrons in the magnetic ribbons around the plasma bubbles are heated by the betatron mechanism to form the thermal part of electron energy spectra. At the same time, part of electrons are trapped and bounce between the two approaching plasma bubbles, and get accelerated mainly by the convective electric field associated with the plasma bubbles, which means that the non-thermal part of electron energy spectra is produced due to Fermi mechanism. When the expanding speed of plasma bubbles is sufficiently large, the non-thermal electrons form power-law spectra. In the late reconnection stage, electrons can be further accelerated mainly by the reconnection electric field in the diffusion region. Different from Totorica et $a .^{41}$, where the reconnection electric field is considered to the main mechanism to produce energetic electrons, we find that Fermi mechanism, as well as the reconnection electric field, plays an important role, espically in the squeezing stage.

Magnetic reconnection experiments in laser-produced plasma have been recently conducted, and energetic electrons are measured in these experiments. ${ }^{37}$ These energetic electrons can reach $\mathrm{MeVs}$ with a power-law spectrum, and the index is measured to be about 6.5. Here, in this paper, our 2-D PIC simulations show that, when the expanding speed of plasma bubbles is sufficiently large, the formed energy spectra of non-thermal electrons have a power-law distribution, which are accelerated in the squeezing and reconnection stages mainly by the Fermi mechanism and reconnection electric field, respectively. The index the power-law spectrum is around 5.7 to 6.0 ( the expanding speed of the plasma bubbles $V_{0} / c_{s}=3-6$ ), which is consistent with the experimental observations. ${ }^{37}$ Our results also imply that the production of energetic electrons with a power-law distribution cannot be considered as a signature of magnetic reconnection between two expanding plasma bubbles with a high speed, because such energetic electrons with a power-law distribution can also be generated from the process of strong squeezing between two plasma bubbles only. 


\section{ACKNOWLEDGMENTS}

This work was supported by 973 Program (2013CBA01503), the National Science Foundation of China (Grant Nos. 41331067, 41527804, 41121003, and 11220101002), and the Key Research Program of Frontier Sciences, CAS (QYZDJ-SSW-DQC010).

\section{REFERENCES}

${ }^{1}$ R. G. Giovanelli, Nature 158, 81 (1946)

${ }^{2}$ S. Tsuneta, Astrophys. J. 456, 840 (1996)

${ }^{3}$ D. N. Baker, T. I. Pulkkinen, V. Angelopoulos, W. Baumjohann, and R. L. McPherron, J. Geophys. Res. 101, 12975, doi:10.1029/95JA03753 (1996)

${ }^{4}$ T. Nagai, M. Fujimoto, Y. Saito, S. Machida, T. Terasawa, R. Nakamura, T. Yamamoto, T. Mukai, A. Nishida, and S. Kokubun, J. Geophys. Res. 103, 4419, doi:10.1029/97JA02190 (1998)

${ }^{5}$ V. Angelopoulos, J. P. McFadden, D. Larson, C. W. Carlson, S. B. Mende, H. Frey, T. Phan, D. G. Sibeck, K. H. Glassmeier, U. Auster, E. Donovan, I. R. Mann, I. J. Rae, C. T. Russell, A. Runov, X. Z. Zhou and L. Kepko, Science 321931 (2008)

${ }^{6}$ J. A. Wesson, Nucl. Fusion 30, 2545 (1990)

${ }^{7}$ M. Yamada, F. M. Levinton, N. Pomphrey, R. Budny, J. Manickam, and Y.

Nagayama, Phys. Plasmas 1, 3269 (1994)

${ }^{8}$ R. P. Lin and H. S. Hudson, Sol. Phys. 17412 (1971)

${ }^{9}$ R. P. Lin, S. Krucker, G. J. Hurford, D. M. Smith, H. S. Hudson, G. D. Holman, R. A. Schwartz, B. R. Dennis, G. H. Share, R. J. Murphy, A. G. Emslie, C. Johns-Krull and N. Vilmer, Astrophys. J. 595 L69 (2003)

${ }^{10}$ M. Hoshino, T. Mukai, T. Terasawa, and I. Shinohara, J. Geophys. Res. 106, 25979, doi:10.1029/2001JA900052 (2001)

${ }^{11}$ X. R. Fu, Q. M. Lu, and S. Wang, Phys. Plasmas 13, 012309 (2006)

${ }^{12}$ P. L. Pritchett, Geophys. Res. Lett. 33 L13104(2006)

${ }^{13}$ C. Huang, Q. M. Lu, and S. Wang, Phys. Plasmas 17, 072306 (2010) 
${ }^{14}$ R. S. Wang, Q. M. Lu, C. Huang, and S. Wang, J. Geophys. Res. 115, A01209, doi:10.1029/2009JA014553 (2010)

${ }^{15}$ M. Y. Wu, C. Huang, Q. M. Lu, M. Volwerk, R. Nakamura, Z. Vörös, T. L. Zhang, and S. Wang, J. Geophys. Res. 120, 6320, doi:10.1002/2015JA021165 (2015)

${ }^{16}$ H. S. Fu, Y. V. Khotyaintsev, M. Andre, and A. Vaivads, Geophys. Res. Lett. 38 L16104 (2011)

${ }^{17}$ M. Y. Wu, Q. M. Lu, M. Volwerk, Z. Vörös, T. L. Zhang, L. C. Shan and C. Huang, J. Geophys. Res. 1184804 (2013)

${ }^{18}$ C. Huang, M. Y. Wu, Q. M. Lu, R. S. Wang and S. Wang, J. Geophys. Res. 1201759 (2015)

${ }^{19}$ S. Lu, Angelopoulos and H. S. Fu, J. Geophys. Res. 1219483 (2016)

${ }^{20}$ J. F. Drake, M. Swisdak, H. Che and M. A. Shay, Nature 443553 (2006)

${ }^{21}$ L. J. Chen, A. Bhattacharjee, P. A. Puhl-Quinn, H. Yang, N. Bessho, S. Imada, S. Mühlbachler, P. W. Daly, B. Lefebvre, Y. Khotyaintsev, A. Vaivads, A. Fazakerley and E. Georgescu, Nat. Phys. 419 (2008)

${ }^{22}$ P. L. Pritchett, Phys. Plasmas 15102105 (2008)

${ }^{23}$ M. Oka, T. D. Phan, S. Krucker, M. Fujimoto and I. Shinohara, Astrophys. J. 714 915 (2010)

${ }^{24}$ H. Y. Wang, Q. M. Lu, C. Huang and S. Wang, Astrophys. J. 82184 (2016)

${ }^{25}$ H. Y. Wang, Q. M. Lu, C. Huang and S. Wang, Phys. Plasmas 24052113 (2017)

${ }^{26}$ W. Daughton, V. Roytershteyn, H. Karimabadi, L. Yin, B. J. Albright, B. Bergen and K. J. Bowers, Nat. Phys. 7539 (2011)

${ }^{27}$ H. Q. Song, Y. Chen, G. Li, X. L. Kong and S. W. Feng, Phys. Rev. X 2021015 (2012)

${ }^{28}$ R. S. Wang, Q. M. Lu, R. Nakamura, C. Huang, A. M. Du, F. Guo, W. L. Teh, M. Y. Wu, S. Lu and S. Wang, Nat. Phys. 12263 (2016)

${ }^{29}$ Y. Zhao, R. S. Wang, Q. M. Lu, A. M. Du, Z. H. Yao and M. Y. Wu, J. Geophys. Res. $12110898(2016)$

${ }^{30}$ F. Guo, H. Li, W. Daughton and Y. H. Liu, Phys. Rev. Lett. 113155005 (2014)

${ }^{31}$ P. M. Nilson, L. Willingale, M. C. Kaluza, C. Kamperidis, S. Minardi, M. S. Wei, P. 
Fernandes, M. Notley, S. Bandyopadhyay, M. Sherlock, R. J. Kingham, M. Tatarakis, Z. Najmudin, W. Rozmus, R. G. Evans, M. G. Haines, A. E. Dangor and K. Krushelnick, Phys. Rev. Lett. 97255001 (2006)

${ }^{32}$ P. M. Nilson, L. Willingale, M. C. Kaluza, C. Kamperidis, S. Minardi, M. S. Wei, P. Fernandes, M. Notley, S. Bandyopadhyay, M. Sherlock, R. J. Kingham, M. Tatarakis, Z. Najmudin, W. Rozmus, R. G. Evans, M. G. Haines, A. E. Dangor and K. Krushelnick, Phys. Plasmas 15092701 (2008)

${ }^{33}$ L. Willingale, P. M. Nilson, M. C. Kaluza, A. E. Dangor, R. G. Evans, P. Fernandes, M. G. Haines, C. Kamperidis, R. J. Kingham, C. P. Ridgers, M. Sherlock, A. G. R. Thomas, M. S. Wei, Z. Najmudin, K. Krushelnick, S. Bandyopadhyay, M. Notley, S. Minardi, M. Tatarakis and W. Rozmus, Phys. Plasmas 17043104 (2010)

${ }^{34}$ C. K. Li, F. H. Séguin, J. A. Frenje, J. R. Rygg, R. D. Petrasso, R. P. J. Town, P. A. Amendt, S. P. Hatchett, O. L. Landen, A. J. Mackinnon, P. K. Patel, M. Tabak, J. P. Knauer, T. C. Sangster and V. A. Smalyuk, Phys. Rev. Lett. 99015001 (2007)

${ }^{35}$ M. J. Rosenberg, C. K. Li, W. Fox, I. Igumenshchev, F. H. Séguin, R. P. J. Town, J. A. Frenje, C. Stoeck1, V. Glebov, R. D. Petrasso, Nat. Commun. 66190 (2015)

${ }^{36}$ J. Y. Zhong, Y. T. Li, X. G. Wang, J. Q. Wang, Q. L. Dong, C. J. Xiao, S. J. Wang, X. Liu, L. Zhang, L. An, F. L. Wang, J. Q. Zhu, Y. Gu, X. T. He, G. Zhao and J. Zhang, Nat. Phys. 6984 (2010)

${ }^{37}$ Q. L. Dong, S. J. Wang, Q. M. Lu, C. Huang, D. W. Yuan, X. Liu, X. X. Lin, Y. Li, H. G. Wei, J. Y. Zhong, J. R. Shi, S. E. Jiang, Y. K. Ding, B. B. Jiang, K. Du, X. T. He, M. Y. Yu, C. S. Liu, S. Wang, Y. J. Tang, J. Q. Zhu, G. Zhao, Z. M. Sheng and J. Zhang, Phys. Rev. Lett. 108215001 (2012)

${ }^{38}$ W. Fox, A. Bhattacharjee and K. Germaschewski, Phys. Rev. Lett. 106215003 (2011)

${ }^{39}$ S. Lu, Q. M. Lu, Q. L. Dong, C. Huang, S. Wang, J. Q. Zhu, Z. M. Sheng and J. Zhang, Phys. Plasmas 20112110 (2013)

${ }^{40}$ K. Huang, C. Huang, Q. L. Dong, Q. M. Lu, S. Lu, Z. M. Sheng, S. Wang and J. Zhang, Phys. Plasmas 24041406 (2017)

${ }^{41}$ S. R. Totorica, T. Abel and F. Fiuza, Phys. Rev. Lett. 116095003 (2016) 
${ }^{42}$ S. Lu, Q. M. Lu, F. Guo, Z. M. Sheng, H. Y. Wang and S. Wang, New J. Phys. 18 013051 (2016)

${ }^{43}$ G. Fiksel, W. Fox, Bhattacharjee, Barnak, P. Y. Chang, K. Germaschewski, S. X. Hu and P. M. Nilson Phys. Rev. Lett 113, 105003 (2014)

${ }^{44}$ S. Lu, Q. M. Lu, C. Huang, Q. L. Dong, J. Q. Zhu, Z. M. Sheng, S. Wang and J.

Zhang, New J. Phys. 16083021 (2014)

${ }^{45}$ Z. Xu, B. Qiao, H. X. Chang, W. P. Yao, S. Z. Wu, X. Q. Yan, C. T. Zhou, X. G. Wang, and X. T. He, Phys. Rev. E 93, 033206, 2016. 


\section{FIGURE CAPTIONS}

Figure.1 The magnetic field $B / B_{0}$ and out-of-plane electron current density $J_{e z} / e n_{0} v_{A}$ at $\Omega_{i} t=0,0.3,0.55$ and 0.75 in AP case (a) and P case (b). The expanding speed is $V_{0} / c_{s}=5$.

Figure.2 Time evolution of the reconnected magnetic flux $\Phi_{B} / B_{0} d_{i}$, the out-of-plane electric field $E_{z} / v_{A} B_{0}$ at $(x, y)=(0,0)$, and the maximum of the upstream magnetic field $B_{\max } / B_{0}$ in AP case (blue full line). The maximum of the upstream magnetic field $B_{\max } / B_{0}$ in P case (blue dash line) is also plotted. Here, the expanding speed is $V_{0} / c_{s}=5$.

Figure.3 Electron energy spectra in AP and P cases for different expanding speeds $V_{0} / c_{s}=$ (a) 2 ,

(b) 3, (c) 4, (d) 5 and (e) 6. The spectra at left column corresponding to those in AP cases, while the right column corresponding to those in $\mathrm{P}$ cases. The black lines represent the initial spectra, the blue lines are the spectra when the maximum of upstream magnetic field reaches its peak in AP cases, and the red lines are the spectra when the flux of non-thermal electrons reaches its peak in AP cases. The blue dotted and red lines corresponds to the Maxwellian distributions fitted from the blue and red lines, respectively.

Figure.4 The relation between the perpendicular temperature of electrons and the average enhancement of magnetic field at the ribbon of the plasma bubbles in AP case at the times when the maximum of upstream magnetic field reaches its peak. From left to right, the five diamond refer to the cases where $V_{0} / c_{s}=2,3,4,5$ and 6 respectively. The full line represents $T_{\perp} / T_{0}=B_{\text {ave }} / B_{0}$

Figure.5 (a) The trajectory of a non-thermal electron produced by Fermi mechanism during $\Omega_{i} t=0$ and 0.6 in AP case, the expanding speed is $V_{0} / c_{s}=5$, the magnetic field lines and the out-of-plane electric field at $\Omega_{i} t=0.3$ are plotted for reference. (b) The electron's energy versus its position in y direction. The color of the trajectory also represents the electron energy. (c) The black line shows the evolution of the electron energy, the blue and red lines show the works done by convective electric field $-\mathbf{u}_{i} \times \mathbf{B}$ (where $\mathbf{u}_{i}$ is the ion bulk velocity) and non-ideal electric 
field $\mathbf{E}+\mathbf{u}_{i} \times \mathbf{B}$ respectively. (d) The electron trajectory in $(z, y)$ plane, and $z$ is calculated by $\int_{0}^{\tau} v_{z} d t$. The red, green and black full diamonds represent $\Omega_{i} t=0.05,0.2$ and 0.58 respectively.

Figure.6 (a) The trajectory of a non-thermal electron produced by Fermi acceleration during $\Omega_{i} t=0$ and 0.6 in P case, the expanding speed is $V_{0} / c_{s}=5$, the magnetic field lines and the out-of-plane electric at $\Omega_{i} t=0.3$ are plotted for reference. (b) The electron's energy versus its position in y direction. The color of the trajectory also represents the electron energy. (c) The black line shows the evolution of the electron energy, the blue and red lines show the works done by convective electric field $-\mathbf{u}_{i} \times \mathbf{B}$ and non-ideal electric field $\mathbf{E}+\mathbf{u}_{i} \times \mathbf{B}$, respectively. (d) The electron trajectory in $(z, y)$ plane, and $z$ is calculated by $\int_{0}^{\tau} v_{z} d t$. The red, green and black full diamonds represent $\Omega_{i} t=0.05,0.2$ and 0.58 respectively.

Figure.7 The initial energy distributions of electrons whose energy exceed $0.8 m_{e} c^{2}$ at $\Omega_{i} t=$ 0.6 (at the end of squeezing stage) in AP case (a) and P case (b), and it is denoted with red color. The initial electron energy spectra are plotted for reference (black color) and the energy distributions of these electrons at $\Omega_{i} t=0.6$ are plotted at the top right corner of each subgraph. The expanding speed is $V_{0} / c_{s}=5$.

Figure.8 (a) The trajectory of a non-thermal electron produced at the reconnection stage during $\Omega_{i} t=0$ and 0.8 in AP case, the expanding speed is $V_{0} / c_{s}=5$, the magnetic field lines and the out-of-plane electric field at $\Omega_{i} t=0.7$ are plotted for reference. (b) The electron's energy versus its position in y direction. The color of the trajectory also represents the electron energy. (c) The black line shows the energy evolution of this electron, the blue and red lines show the works done by the convectiv electric field $-\mathbf{u}_{i} \times \mathbf{B}$ and non-ideal electric field $\mathbf{E}+\mathbf{u}_{i} \times \mathbf{B}$ respectively. (d) The electron trajectory in $(z, y)$ plane, and $z$ is calculated by $\int_{0}^{\tau} v_{z} d t$. The red, green and black full diamonds represent $\Omega_{i} t=0.1,0.6$ and 0.77 respectively.

Figure.9 (a) The energy distributions of electrons at $\Omega_{i} t=0.6$, whose energy exceeds $1.0 m_{e} c^{2}$ at $\Omega_{i} t=0.79$ (the flux of non-thermal electrons reaches maximum) in AP case, and it is denoted 
with red color. (b) The energy distribution of electrons at $\Omega_{i} t=0.6$, whose energy exceeds $0.8 m_{e} c^{2}$ at $\Omega_{i} t=0.79$ in P case, and it is denoted with red color. The electron energy spectra at $\Omega_{i} t=0.6$ are plotted for reference (black color) and the energy distributions of these electrons at $\Omega_{i} t=0.79$ are plotted at the top right corner of each subgraph. The expanding speed is $V_{0} / c_{s}=$ 5. 
(a)

(b)

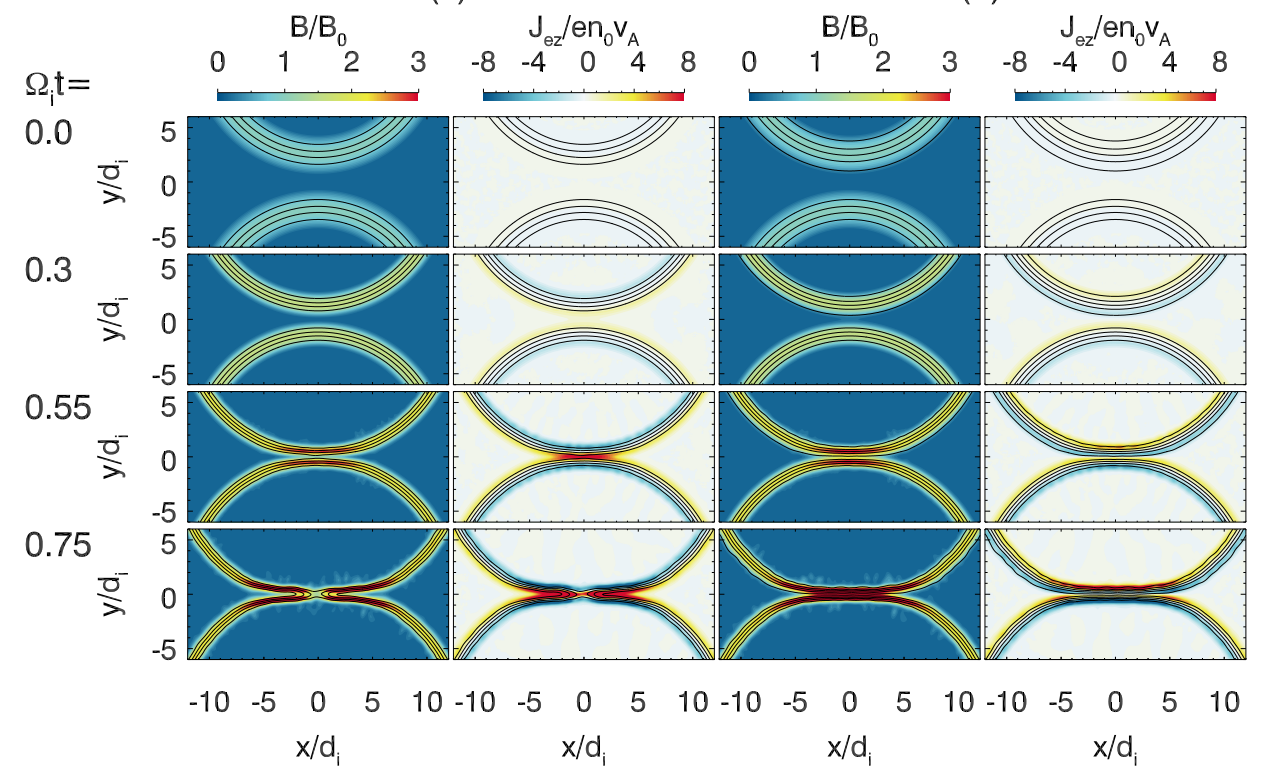




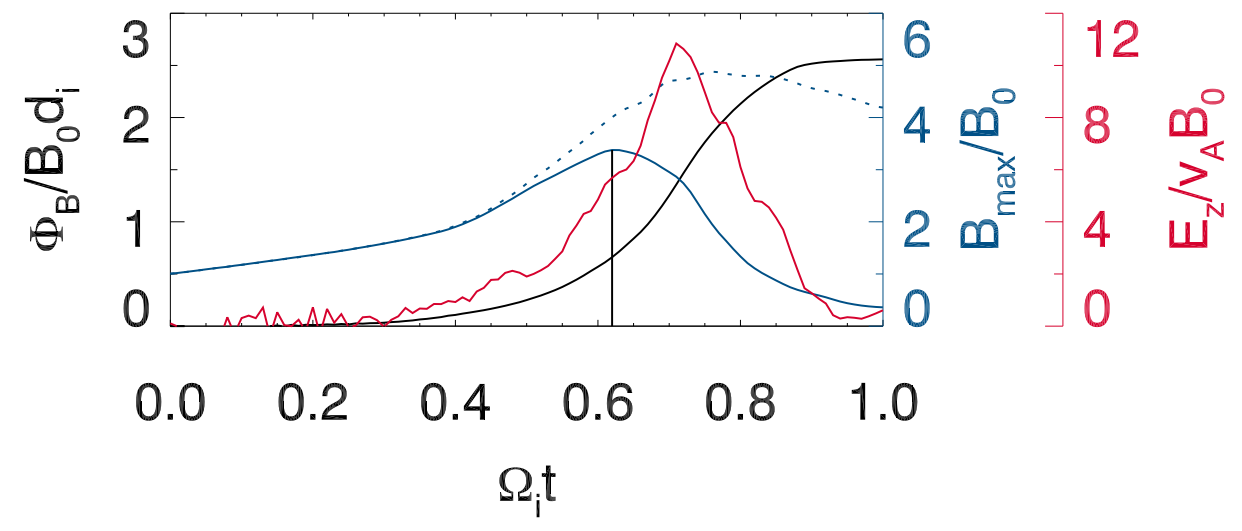




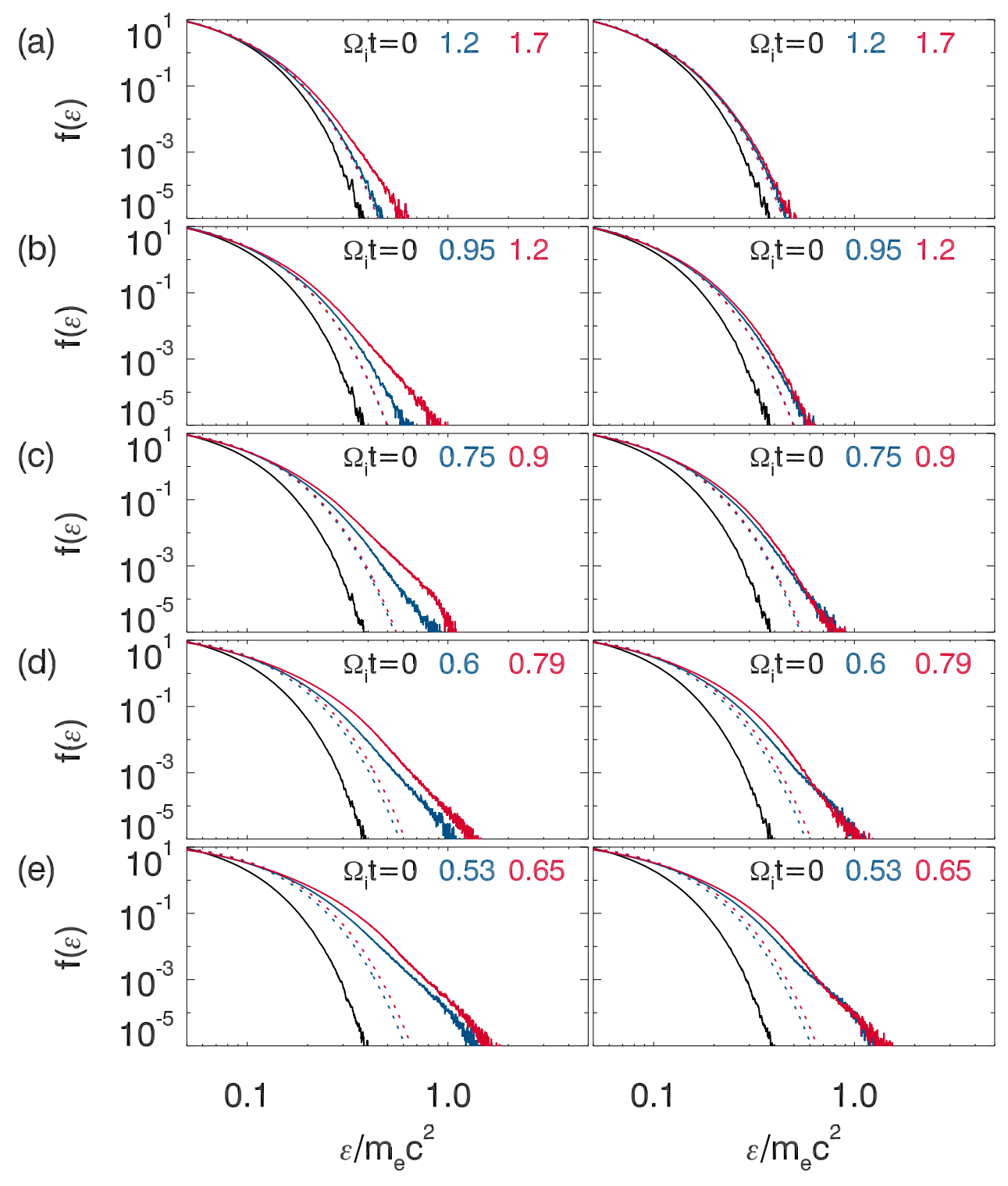




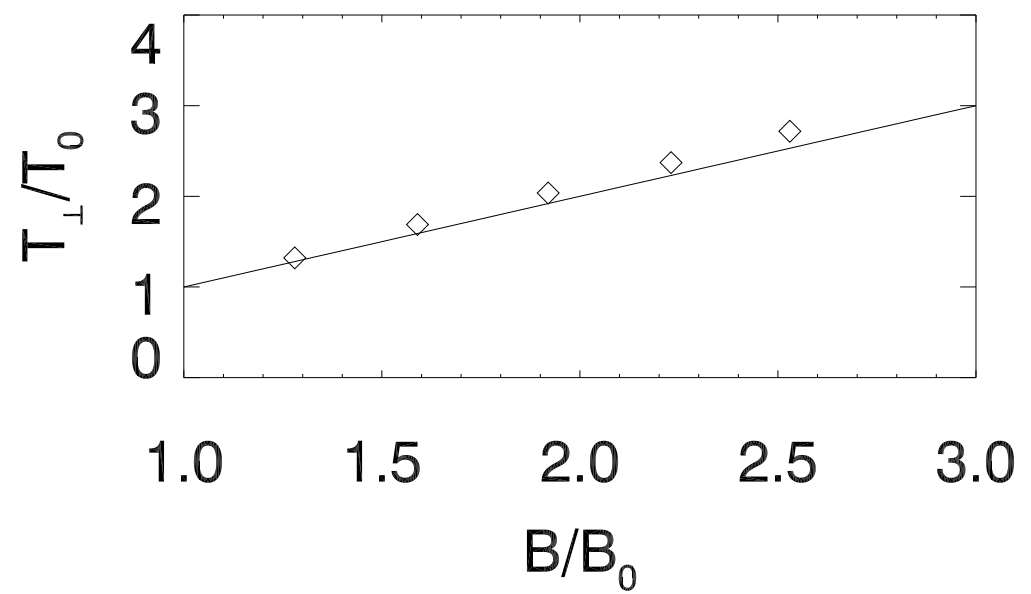



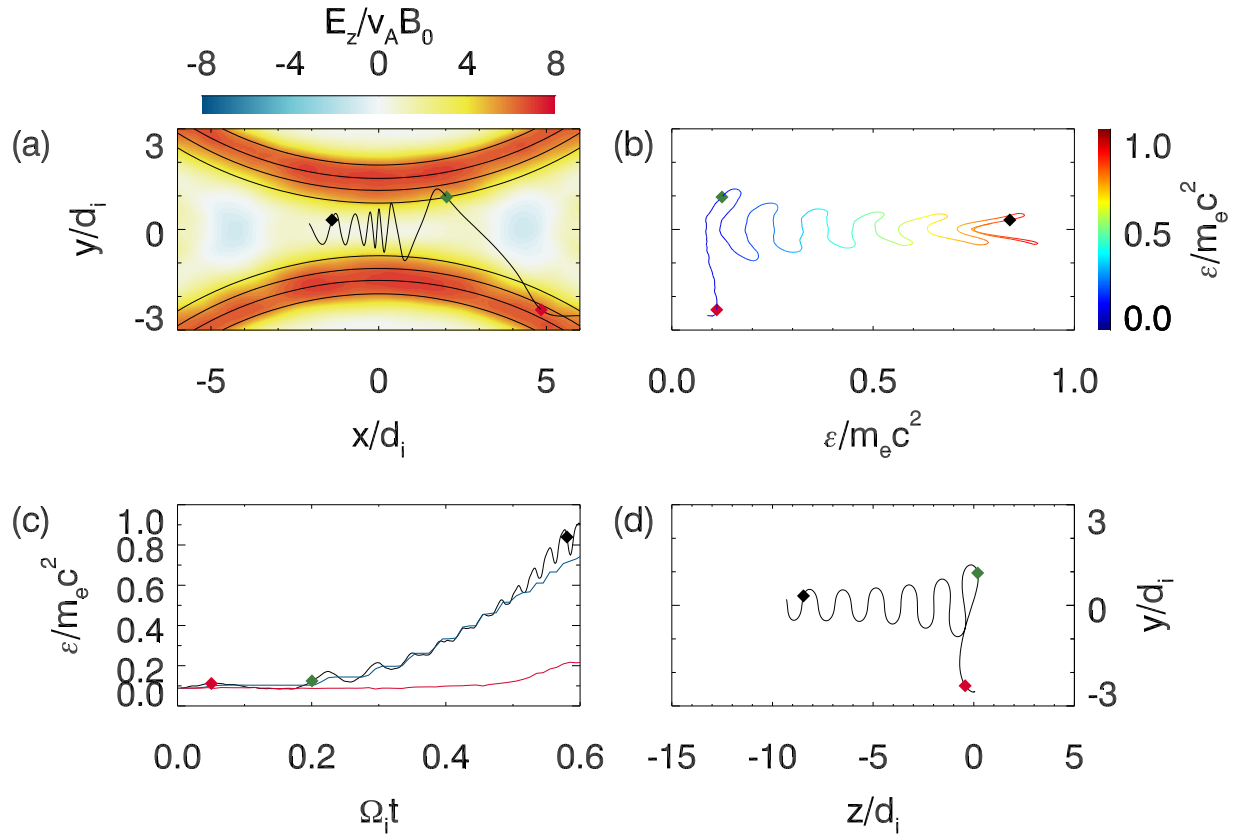

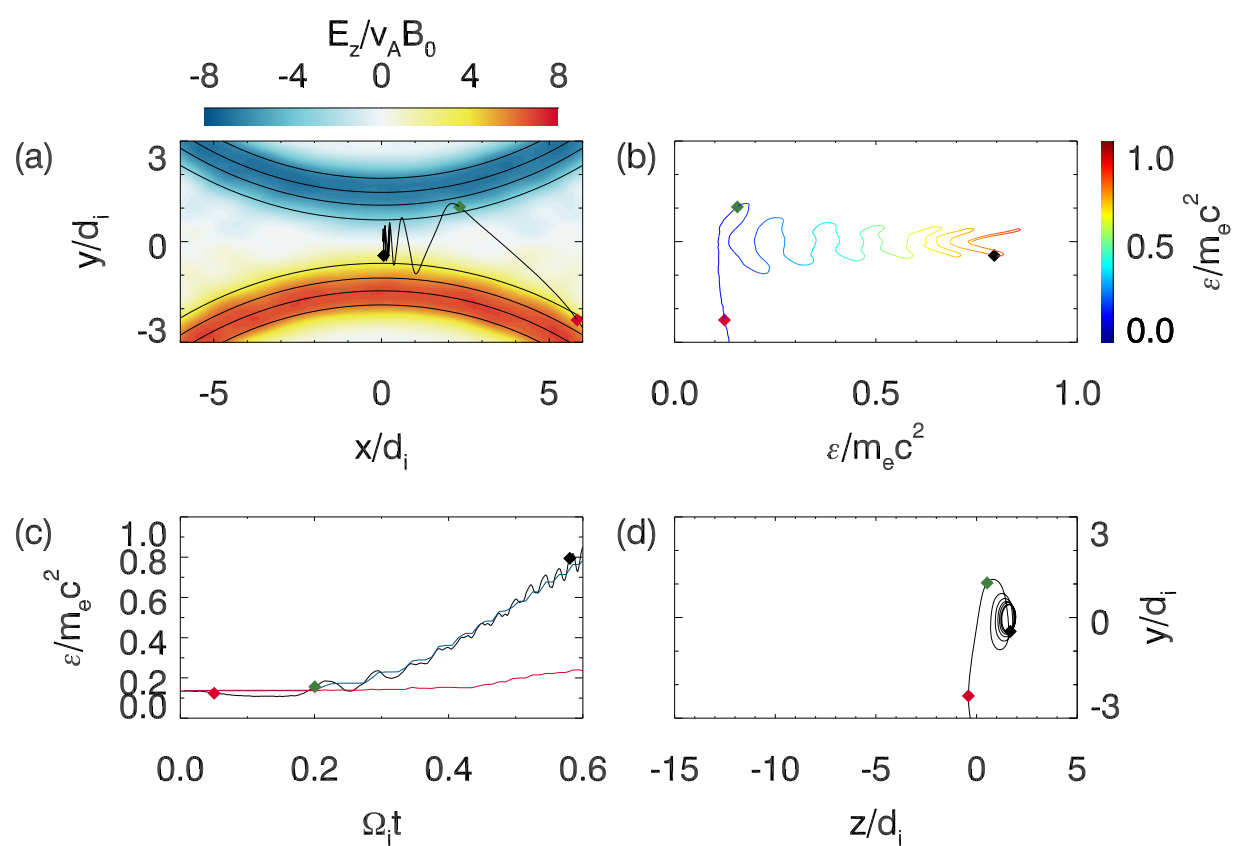


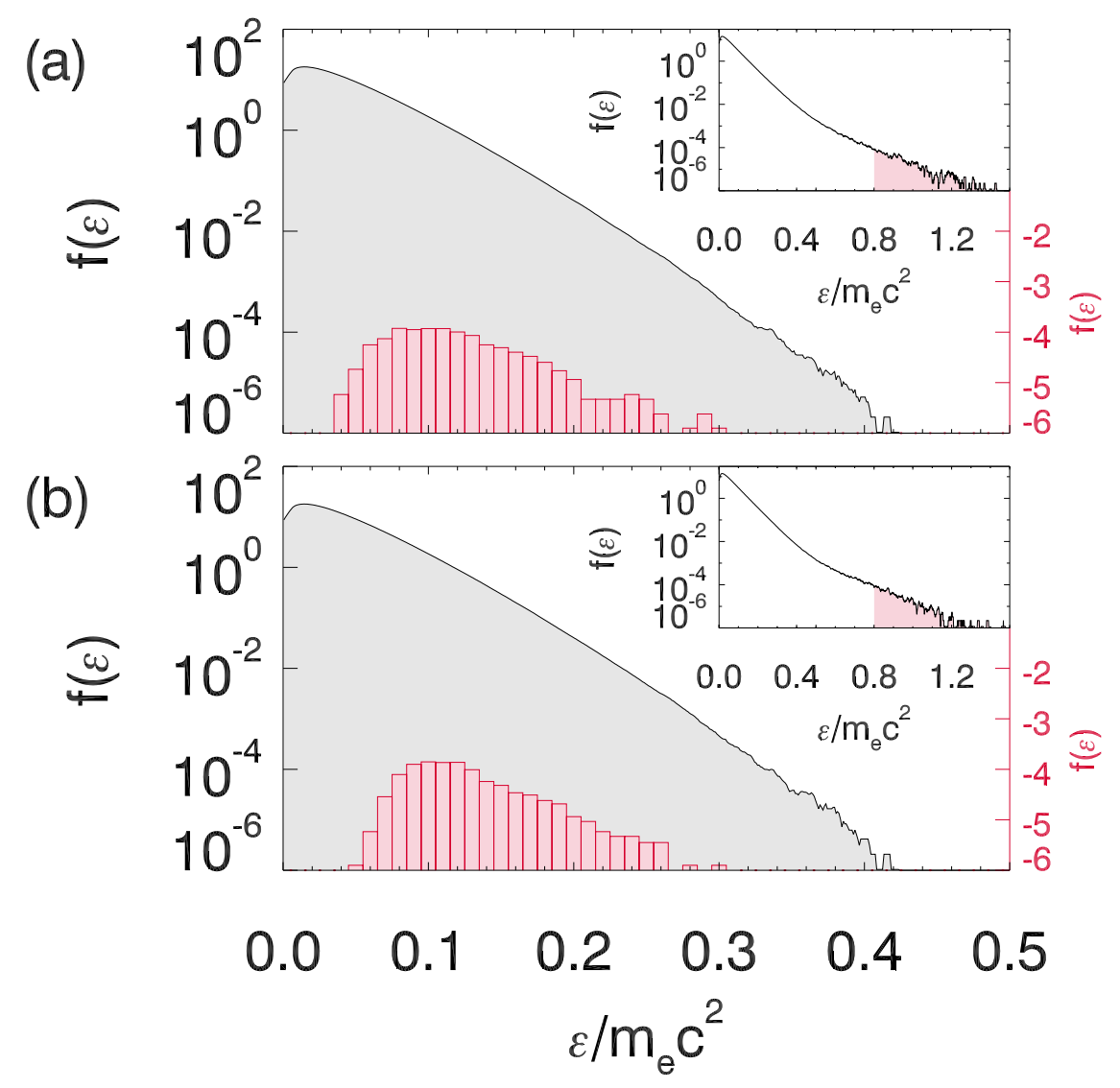




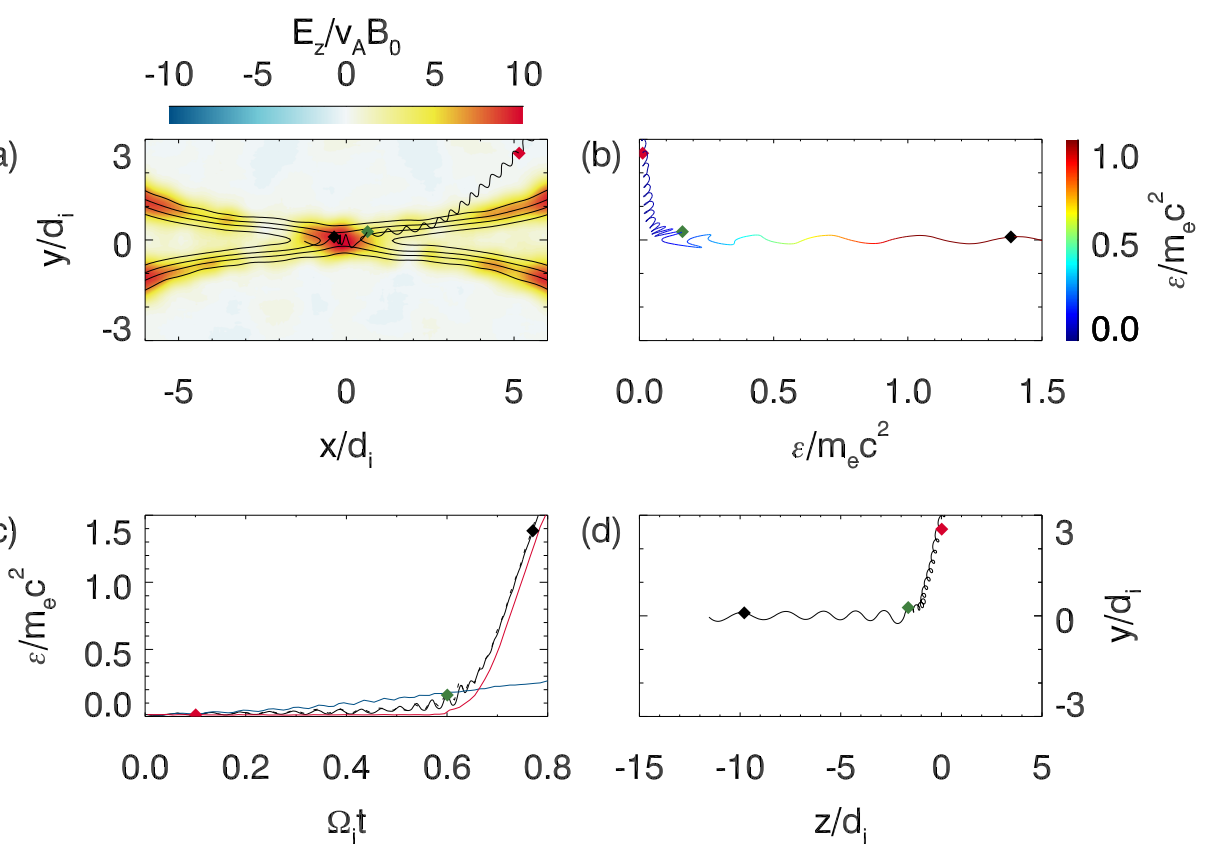



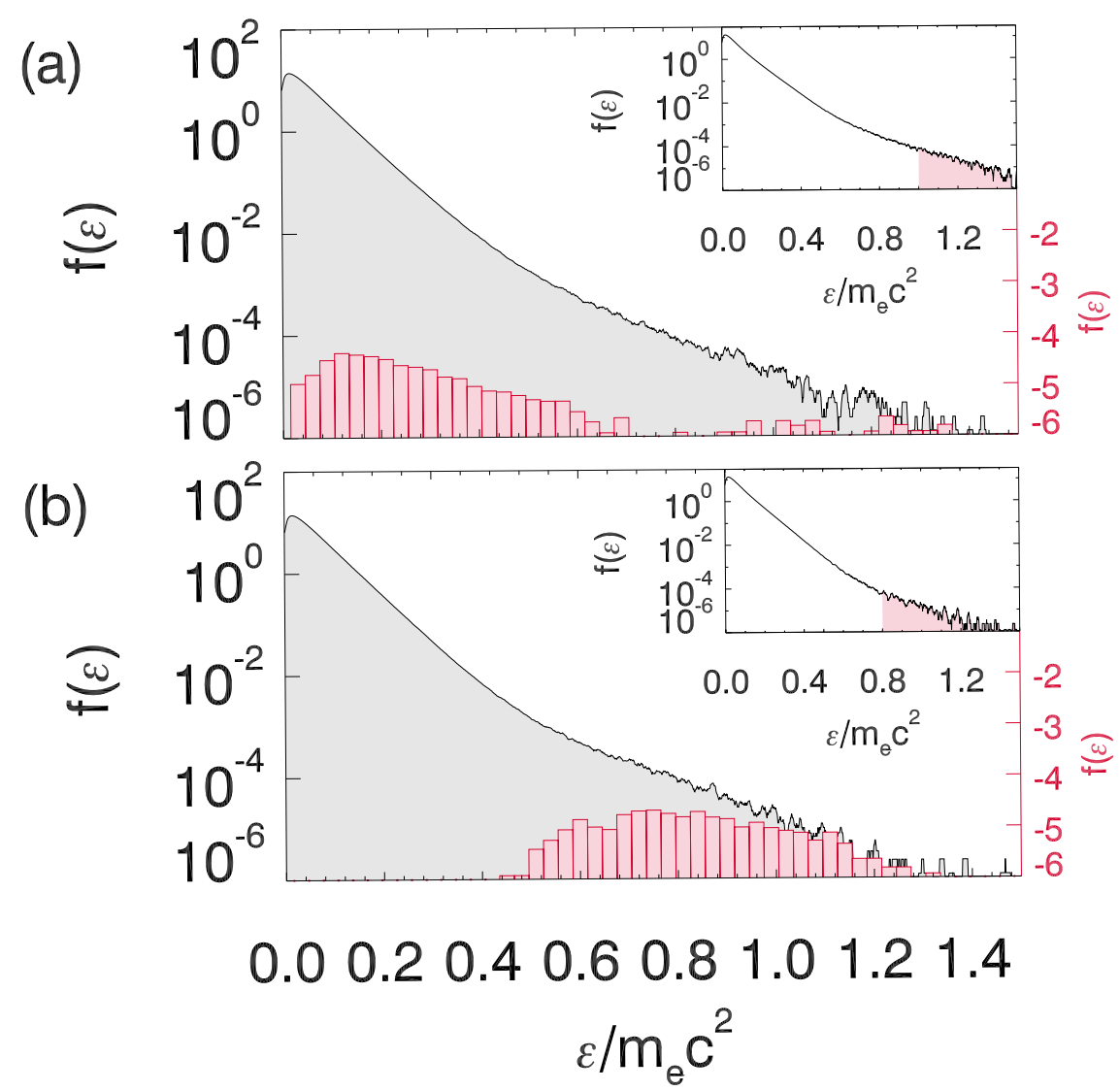